\title{
The Insidious Problem of Fatigue in Medical Imaging Practice
}

\author{
Bruce I. Reiner • Elizabeth Krupinski
}

Published online: 6 December 2011

(C) Society for Imaging Informatics in Medicine 2011

\section{Introduction}

Fatigue represents a temporary inability to respond to a situation due to inadequate recuperation from overactivity; which can manifest in mental, emotional, or physical forms [1]. Occupational fatigue has been identified as a contributing factor in numerous catastrophic events including the Three Mile Island and Chernobyl nuclear reactor meltdowns, the Challenger Space Shuttle disaster, The Bhopal Union Carbide plant explosion, and the grounding and resulting oil spill of the Exxon Valdez oil tanker $[2,3]$. In medicine, fatigue has been well documented as a source of medical errors, exacerbated by the continuous (i.e., around the clock) requirements for service delivery as well as associated disruption of circadian rhythms [4, 5]. Other sources of medical error that could be exacerbated by fatigue include excessive workload, cognitive overload, imperfect information processing, poor communication, and flawed decision making [6].

One can argue that all of these sources of medical error are ubiquitous in the current healthcare practice, and steadily rising as service demands and quality expectations continue to escalate. As reimbursements trend downwards, healthcare

B. I. Reiner $(\bowtie)$

Department of Radiology,

Veterans Affairs Maryland Healthcare System,

10 North Greene Street,

Baltimore, MD 21201, USA

e-mail: breiner1@comcast.net

E. Krupinski

Department of Radiology, Psychology and the Arizona

Telemedicine Program, University of Arizona,

1609 N Warren, Bldg 211, Rm. 112,

Tucson, AZ 85724, USA providers attempt to compensate by increasing their individual and collective practice productivity. While computerized medical technologies offer the potential to improve workflow and productivity, there is a theoretical point in which increasing productivity becomes offset by potential quality deficiencies. At the same time, healthcare consumers are placing increasing demands on providers for access to healthcare data, collaborative decision making, and quality accountability measures [7-9]. The collective stressors of worsening healthcare economics, increased workload, and heightened quality concerns serve as inevitable source of fatigue and stress on healthcare providers. These in turn can further compromise productivity and quality deliverables. If this cycle of perpetual occupational fatigue is to be successfully addressed and circumvented, it is first essential that the healthcare community identify the sources, create proactive mechanisms for objective data collection and analysis, and develop effective countermeasures.

\section{Unique Attributes of Fatigue in Radiology}

All healthcare professionals are subject to fatigue, and it tends to be progressive in nature, as service providers (both individual and institutional) are expected to "do more with less." As human resources are continuously constrained by economic pressures, technology is expected to compensate, but in itself can contribute to occupational fatigue and stress. Technology-induced fatigue is of particular importance to the radiology community due to the profession's dependence upon technology, continuous evolutionary changes in technology refinement and development, and lack of technology adaptability to end-user attributes and preferences $[10,11]$. 
The current medical imaging and information system technology development model of "one size fits all" causes radiology service providers to operate in a working environment of relative inflexibility, which can constrain workflow, productivity, and end-user acceptance [10]. The irony is that while technology can often serve as a fatigue precipitant, it is also looked upon as a potential solution to fatigue reduction, quality improvement, and increased productivity. In order to accomplish this task, we must better understand both the common and unique attributes of radiology workflow relative to other medical specialties and strive to create innovative solutions that are both customizable to the individual user but still broad-based in nature.

Unlike most other clinicians whose workflow is largely patient-centric, radiologist workflow is almost exclusively tied to the computer, with the exception of interventional, mammographic, and fluoroscopic procedures. While a great deal of other clinicians' time is spent "hands on," the radiologist's time is instead "eyes on," with the workflow of a diagnostic radiologist essentially confined to the human-computer interface. As the volume and complexity of medical imaging data continue to increase, radiologists are faced with progressive occupational fatigue, which takes on an additional component in the forms of eye strain and visual fatigue [12]. Radiologist eye strain and visual fatigue has been shown to adversely affect radiologist productivity and diagnostic accuracy by contributing to perceptual errors, performance errors, decreased reaction time, and even burn out [13].

In addition to traditional and visual sources of occupational fatigue, "decision fatigue" is increasingly documented; which is the result of continuous and prolonged decision making $[14,15]$. It exacts a subconscious toll on your brain, which then responds by taking short cuts to ease mental strain. These short cuts often lead to poor and/or inaccurate decisions being made. As described by the social psychologist Roy Baumeister, decision fatigue is the result of ego depletion, and can result in either careless decisions or decision paralysis. This theory leads one to hypothesize that medical error rates increase later in the day (or duration of the defined work shift), and are especially common in workers who are tasked with repetitive and prolonged decision making.

While all medical imaging professionals are prone to decision fatigue; those of greatest susceptibility are those working prolonged shifts, off hours (with an associated tendency to sleep deprivation), and performing highvolume and high-complexity tasks [16-18]. Given these risk factors, perhaps the segment of the radiologist community at greatest risk for fatigue would be those practicing teleradiology. By its very nature, teleradiology providers practice in a high-volume environment where individual reimbursement is directly tied to output. At the same time, many teleradiology providers operate in a "continuous queue" where exam volumes are continuously replenished and the availability of studies to be read is essentially never ending. In many ways, a busy radiology practice can be likened to an assembly line, where individual workers are paid on a "per piece" basis, similar to the garment industry workers a century ago. The primary difference in this situation is that the tasks being performed are far from mundane and simplistic in nature, but instead involve human lives. As a result, it is imperative that reduction of medical errors always remain the highest priority for service providers (and payers) and innovation focus on methods to identify and intervene when occupational fatigue adversely affects clinical outcomes.

\section{The Relationship Between Cognitive Overload, Stress, and Human Errors}

Human error can be viewed in two different ways: the person approach and the system approach. The person approach focuses on individuals' errors, blaming them for forgetfulness and/or inattention. The system approach concentrates on the conditions and technologies with which collective individuals work and attempts to create strategies to avert and mitigate errors and their resulting effects [19].

While both approaches are valid and applicable to medicine, the system approach theoretically represents a greater opportunity for process improvement due to the organizational reach of systematic intervention and technology innovation to combat human errors and their resulting adverse clinical outcomes. The system approach recognizes that error is the not the monopoly of an unfortunate few, but instead is often recurrent in nature. Relevant examples where recurrent medical errors and patient safety have been addressed through systematic analysis and intervention include mandatory timeouts for surgical/interventional procedures, integration of computerized decision support technologies for diagnosis and interpretation, and incorporation of Six Sigma performance-improvement strategies in the intensive care unit [20-22].

While the system approach to human error offers theoretical benefits in achieving organizational improvement, the person approach is beneficial in understanding the role of inter and intra-radiologist variability on performance. Each individual has been shown to possess a critical point of information processing, and if it is exceeded leads to diminished performance (i.e., individual information processing effectiveness). This critical point in individual information processing effectiveness is in part determined by an individual's personality [23]. The net 
effect of these observations is that both the person and system approaches are critical in understanding the role fatigue plays in human error, and resulting solutions must take both approaches into account.

A wide variety of causes have been reported to be associated with human errors in medicine including fatigue, workload, cognitive overload, imperfect information processing, poor communication, and flawed decision making [6]. Of these various causes, the two which are of particular relevance to this discussion are fatigue and cognitive overload, which are inextricably tied to one another [24, 25].

Cognitive overload can be thought of as exceeding human information processing capacity, which was first described by Miller in 1956 [26]. The number of variables that can be integrated into a single cognitive representation is a major constraint on cognitive and neuropsychological processes [27]. Unfortunately, in contemporary radiology practice, the number and complexity of variables requiring analysis for accurate interpretation of a medical imaging study continues to increase, as imaging datasets increase in size, number, and complexity.

\section{Evolutionary Pressures in Radiology Practice Affecting Fatigue}

The trend for radiology practice over the past decade has been one of increasing exam volume, exam size and complexity, and expectations for quality deliverables [28]. These increasing work demands on the radiologist community have likely contributed to increased visual fatigue [29]. A number of technical factors have also been reported to contribute to visual fatigue including workstation design, monitor resolution, brightness and contrast, image refresh rates, screen flicker and glare, and working distances and angles [12].

While workstation design and monitor optics can be improved upon through product improvement, the trend towards information (data) overload will continue to be exacerbated as the size, complexity, and visualization options for medical imaging datasets continues to expand. Data overload is not restricted to medical imaging data alone, but also includes clinical data which is fundamental to rendering an accurate and definitive diagnosis. As the breadth and depth of medical data expands (e.g., molecular imaging, genomic data), healthcare providers are expected to routinely access and analyze these data in order to improve medical diagnosis and treatment. Unfortunately, technology has not kept up with these increased workflow demands. The lack of information system interoperability, automated data extraction, and end-user customization features leave radiologists and other clinicians in a quandary as to the how these data can be utilized without adversely affecting productivity.
At the same time, medical imaging is experiencing commoditization pressures, which place greater emphasis on cost in service provider selection [30]. In the absence of standardized quality metrics to differentiate service deliverables, many radiologists attempt to compensate to deteriorating economic conditions by increasing exam volumes in order to maintain revenue. The net effect of these economic, technological, and workload pressures are a continued escalation in stress and fatigue on the radiologist community, which has the potential to negatively impact performance and emotional well-being. It is therefore incumbent upon the research, clinical, and technology provider communities to devote greater resources to the scientific study of fatigue and creation of new technologies to combat it.

\section{Conclusion}

By directly integrating fatigue measurement tools into workstation design and routine workflow, one could create a mechanism for objectively quantifying fatigue and determining risk factors specific to both individual and collective radiologist end-users. This objective fatigue data can in turn be correlated with performance metrics tied to productivity, workflow, and quality in order to create customizable fatigue profiles that can take into account both fixed and dynamic attributes of each radiologist or technologist, as well as the technology being used. The analysis of these data can in turn be used to derive "best-practice" clinical guidelines, comparative technology assessment, and creation of new tools and practices for customizable fatigue reduction and performance enhancement. The creation of this proposed technology is less dependent upon technology development than the willingness of the medical imaging community to embrace innovation and change through objective data analysis. In the end, technology and derived data can be beneficial to performance enhancement, as long as they can be customized to the unique needs and preference of each end-user, while also contributing to best practice standards.

\section{References}

1. http://www.osh.govt.nz/catalogue/pdf/stress.pdf [accessed October 3, 2011]

2. Mittler MM, Carskadan MA, Czeisler CA, et al: Catastrophes, sleep, and public policy: consensus report. Sleep 11:100-109, 1988

3. Dinges DF: An overview of sleepiness and accidents. J Sleep Res 4:4-14, 1995

4. Leape LL: Errors in medicine. JAMA 272:1851-1857, 1994

5. Kohn LT, Corrigan J, Donaldson MS: To err is human: building a safer health system. Washington DC: Helmreich RL. On error management: lessons from aviation. BMJ 32:781-785, 2000. National Academy Press 
6. Helmreich RL: On error management: lessons from aviation. BMJ 32:781-785, 2000

7. Hassol A, Walker JM, Kidder D, et al: Patient experiences and attitudes about access to a patient electronic healthcare record and linked web messaging. J Am Med Inform Assoc 11:505-513, 2004

8. Detmer D, Bloomrosen M, Raymond B, et al: Integrated personal health records: transformative tools for consumer-centric care. BMC Med Inform Decis Mak 8:45, 2008

9. Elwyn G, Edwards A, Kinnersley P, et al: Shared decision making and the concept of equipose: the competence of involving patients in healthcare choices. Br J Gen Pract 50:892-897, 2000

10. Reiner B: One size (doesn't) fit all. J Am Coll Radiol 4:567-570, 2008

11. Reiner BI, Siegel EL, Siddiqui K: Evolution of the digital revolution: a radiologist perspective. J Digit Imaging 16:324330, 2003

12. Vertinsky T, Forster B: Prevalence of eye strain among radiologists: influence of viewing variables on symptoms. AJR 184:681-686, 2005

13. Krupinski EA: Medical image perception issues for PACS deployment. Semin Roentgenol 38:231-243, 2003

14. http://www.nytimes.com/2011/08/21/magazine/do-you-suffer-fromdecision-fatigue [accessed 9/28/2011

15. Baumeister RF. "The Psychology of Irrationality", in Brocase, Isabelle and Carrillo Juan D, The Psychology of Economic Decisions: Rationality and Well-Being, pp 1-15

16. Gaba DM, Howard SK: Fatigue among clinicians and the safety of patients. N Engl J Med 347:1249-1255, 2002

17. Veasey S, Rosen R, Barzansky B, et al: Sleep loss and fatigue in residency training: a reappraisal. JAMA 288:1116-1124, 2002

18. MacDonald W: The impact of job demands and workload on stress and fatigue. Australian Psychologist 38:102-117, 2003
19. Reason J: Human error: models and management. BMJ 320:768770, 2000. check volume number

20. Clarke JR, Johnston J, Finley ED: Getting surgery right. Ann Surg 246:395-405, 2007

21. Seiden SC, Barach D: Wrong-side/wrong-site, wrong-procedure, and wrong-patient adverse events. Arch Surg 141:931-939, 2006

22. Bates DW, Gawande AA: Improving safety with information technology. N Engl J Med 348:2526-2534, 2003

23. Seck M, Frydman C, Giambiasi N, et al. Use of a dynamic personality filter in discrete event simulation of human behavior under stress and fatigue. 1st International Conference on Augmented Cognition, July 22-27, 2005 Las Vegas, Nevada

24. Shirom A, Nirel N, Vinokur AM: Overload, autonomy, and burnout as predictors of physicians' quality of care. Journal of Occupational Health Psychology 11:328-342, 2006

25. Krupinski EA, Berbaum KS: The medical image perception society update on key issues for image perception research. Radiology 253:230-233, 2009

26. Miller GA: The magical number seven, plus or minus two: some limits on our capacity for processing information. Psychological Review 63:81-97, 1956

27. Halford GS, Baker R, McCredden JE, Bain JD: How many variables can humans process? Psychological Science 16:70-76, 2005

28. Sunshine JH, Maynard CD: Update on the diagnostic radiology employment market: findings through 2007-2008. J Am Coll Radiol 5:827-833, 2008. and additional references

29. Krupinski EA, Berbaum KS, Caldwell RT, et al: Long radiology workdays reduce detection and accommodation accuracy. J Am Coll Radiol 7:698-704, 2010

30. Reiner B, Siegel E: Decommoditizing radiology. J Am Coll Radiol 3:167-170, 2009 